\title{
On the kinematics of neutral gas in GRB host galaxies
}

\author{
M. Arabsalmani ${ }^{1,2}$, P. Møller ${ }^{1}$, W. Freudling ${ }^{1}$, T. Zafar ${ }^{1}$, J. P. U. \\ Fynbo $^{2}$, L. Christensen ${ }^{2}$ and S. Savaglio ${ }^{3,1}$ \\ ${ }^{1}$ European Southern Observatory, Karl-Schwarzschild-Strasse 2, D-85748 Garching bei \\ Mnchen, Germany \\ email: marabsal@eso.org \\ ${ }^{2}$ Dark Cosmology Centre, Niels Bohr Institute, University of Copenhagen, \\ Juliane Maries Vej 30, 2100 Copenhagen, Denmark \\ ${ }^{3}$ Physics Department, University of Calabria, via P. Bucci, I-87036 Arcavacata di Rende, Italy
}

\begin{abstract}
We analyze a sample of 20 absorption systems intrinsic to long duration GRB host galaxies at $\mathrm{z}>2$ forwhich the metallicities are known. We compare the relation between the metallicity and cold gas velocity width for thissample to that of the Damped Lyman-alpha systems (DLAs) in the sight-lines of quasars (QSOs), and find completeagreement. We then compare the redshift evolution of the velocity-metallicity relation of our sample to that of QSODLAsand find that also GRB hosts favour a late onset of this evolution, around a redshift of $\sim 2.6$. We compute predicted stellar masses for the GRB host galaxies using the prescription determined from QSO-DLAsamples and compare the measured stellar masses for the four hosts where stellar masses have been determinedfrom SED fits. We find excellent agreement and conclude that, on basis of all available data and tests, long durationGRB-DLA hosts and intervening QSO-DLAs are consistent with being drawn from the same underlying population.
\end{abstract}

